\title{
NONEXISTENCE OF GLOBAL SOLUTIONS AND BIFURCATION ANALYSIS FOR A BOUNDARY-VALUE PROBLEM OF PARABOLIC TYPE
}

\author{
C. V. PAO
}

\begin{abstract}
The aim of this paper is to present a bifurcation analysis on the existence of the nonexistence of a global solution for a semilinear parabolic equation and to characterize the local stability and the instability of the corresponding steady-state solutions. The bifurcation result can be described either by a parameter $\lambda$ for a fixed spatial domain $\Omega$ or by varying $\Omega$ for a fixed $\lambda$. The stability analysis gives a result which can be used to determine the stability or instability problem when the system possesses nonintersecting multiple steady-state solutions.
\end{abstract}

1. Introduction. Let $\Omega$ be a bounded domain in $R^{n}$ and $\partial \Omega$ the boundary of $\Omega$. Consider the following initial boundary-value problem:

$$
\begin{gathered}
u_{t}-\nabla \cdot(D(x) \nabla u)=\lambda\left(e^{a u}-b\right) \quad(t>0, x \in \Omega), \\
\beta(\partial u / \partial \nu)+u=0 \quad(t>0, x \in \partial \Omega), \\
u(0, x)=u_{0}(x) \quad(x \in \Omega),
\end{gathered}
$$

where $\lambda, \beta, a, b$ are nonnegative constants with $0 \leqslant b \leqslant 1, D$ is a positive function on $\bar{\Omega}$ (the closure of $\Omega$ ), $\nabla$ is the gradient operator in $\Omega$ and $\partial / \partial \nu$ is the outward normal derivative on $\partial \Omega$. We assume that $\Omega$ is sufficiently smooth, $D$ is continuously differentiable in $\bar{\Omega}$, and $u_{0}$ is continuous nonnegative on $\bar{\Omega}$ and satisfies the boundary condition (1.2). The above system arises in the thermal ignition of mixture of gases and some nonlinear diffusion problems (cf. [6], [8], [9]). The purpose of this paper is to give a bifurcation analysis on the existence and nonexistence of a global solution of the system (1.1)-(1.3), and to characterize the local stability and instability behavior of steady-state solutions. Our bifurcation results can be described either by considering $\lambda$ as a parameter with a fixed domain $\Omega$ or by varying the size of $\Omega$ with a fixed $\lambda$. Specifically, we show for the case of fixed $\Omega$ that as $\lambda$ increases from zero a unique global solution exists until $\lambda=\lambda_{c}$ for some $\lambda_{c}>0$. As soon as $\lambda>\lambda_{c}$ this solution not only grows unbounded but also diverges to $\infty$ in a finite time $T_{\lambda}$ which decreases as $\lambda$ increases. In fact, we obtain an explicit upper bound for the "finite escape time" $T_{\lambda}$. We also show that if a steady-state solution $u_{e}(x)$ exists then it is either asymptotically

Received by the editors August 2, 1976.

AMS (MOS) subject classifications (1970). Primary 35K20, 35B40; Secondary 35K60.

Key words and phrases. Nonexistence of global solution, parabolic equation, local stability and instability, bifurcation analysis.

- American Mathematical Society 1977 
stable or unstable depending solely on $\lambda \exp \left(a u_{e}(x)\right)<\lambda_{c}$ or $>\lambda_{c}$ on $\bar{\Omega}$. This result characterizes the local behavior of a steady-state solution which can be used to determine the stability and instability problem when the system possesses nonintersecting multiple steady-state solutions.

The nonexistence of global solutions for semilinear parabolic equations was discussed in [5], [7], [15]. The papers of [7], [15] are concerned with initialvalue problems with $\Omega=R^{n}$ while the work in [5] treats a special form of (1.1)-(1.3) with $D=a=\lambda=1$ and $\beta=b=0$. It was announced in [5] that if the size of $\Omega$ is sufficiently large then the solution diverges to $\infty$ either as $t \rightarrow \infty$ or in a finite time. In this paper, we obtain a more definite conclusion for the system (1.1)-(1.3). Specifically, we show that there is a domain $\Omega_{c}$ such that the solution actually diverges to $\infty$ in finite time when the size of $\Omega$ is increased beyond the size of $\Omega_{c}$. On the other hand, the existence of positive solutions for the corresponding steady-state solution has recently been investigated in [1], [2], [9], [14]. In particular, the bounds of the steady-state solution given in [2] coupled with our results can be used to obtain detailed information about the stability problem such as stability region and rate of decay of the perturbed solution to the steady-state solution. Our approach to the stability problem uses a similar technique as that in [3].

2. The main results. By a steady-state solution we mean a time-independent solution $u_{e}(x)$ of $(1.1),(1.2)$. In view of the maxium principle any steady-state solution is nonnegative. Notice that if $b=1$ the zero function $u_{e}=0$ is a steady-state solution. Let $\mu_{0}$ be the last eigenvalue and $\psi(x)$ the corresponding eigenfunction of the linear eigenvalue problem

$$
\begin{array}{cc}
\nabla \cdot(D(x) \nabla \phi)+\mu \phi=0 & (x \in \Omega), \\
\beta(\partial \phi / \partial \nu)+\phi=0 & (x \in \partial \Omega) .
\end{array}
$$

It is well known that $\mu_{0}$ is positive and $\psi(x)>0$ in $\Omega$. In fact, if $\beta>0$ then the maximum principle implies that $\psi(x)>0$ on $\bar{\Omega}$ (cf. [12]). We set $\psi_{m}=$ $\min \psi(x)$ on $\bar{\Omega}$ and normalize $\psi(x)$ so that $\max \psi(x)=1$. Notice that $\psi_{m}>0$ when $\beta>0$. Our results for the existence and nonexistence of global solutions are given in the following two theorems.

THEOREM 1. Let $\beta>0$ and let $u_{0}(x) \geqslant \delta \psi(x)$ for some constant $\delta>0$. If $a \lambda \geqslant \mu_{0}$ then there exists a constant $T_{1}<\infty$ such that a unique nonnegative solution $u(t, x)$ to $(1.1)-(1.3)$ exists on $\left[0, T_{1}\right) \times \bar{\Omega}$ and satisifes

$$
\lim _{t \rightarrow T_{1}} \max _{x \in \bar{\Omega}} u(t, x)=\infty \text {. }
$$

In fact, $u(t, x) \geqslant \delta(1-\gamma t)^{-1} \psi(x)$, where $\gamma=\lambda \delta a^{2} \psi_{m} / 2$.

THEOREM 2. Let $b=1$ and let $u_{0}(x) \leqslant \rho \psi(x)$ for a sufficiently small constant $\rho>0$. If $a \lambda<\mu_{0}$ then a unique nonnegative solution $u(t, x)$ to $(1.1)-(1.3)$ exists on $[0, \infty) \times \bar{\Omega}$ and satisfies

$$
\lim _{t \rightarrow \infty} \max _{x \in \bar{\Omega}} u(t, x)=0
$$


Theorem 2 implies that when $b=1$ the zero solution is asymptotically stable. In the case of $b<1$, positive steady-state solutions still exist when $\lambda a$ is sufficiently small (cf. [2], [12]). Our next theorem is to characterize the local stability and instability behavior of any nonnegative steady-state solution.

THEOREM 3. Let $u_{e}(x)$ be any nonnegative steady-state solution. If

$$
\exp \left(a u_{e}(x)\right)<(a \lambda)^{-1} \mu_{0} \quad(x \in \bar{\Omega}),
$$

then there exist positive constants $\rho_{i}, \alpha_{i}, i=1,2$, such that a unique solution $u(t, x)$ to $(1.1)-(1.3)$ exists and satisfies

$$
\begin{aligned}
u_{e}(x)-\rho_{1} e^{-\alpha_{1} t} \psi(x) & \leqslant u(t, x) \\
& \leqslant u_{e}(x)+\rho_{2} e^{-\alpha_{2} t} \psi(x) \quad(t>0, x \in \bar{\Omega})
\end{aligned}
$$

provided that

$$
u_{e}(x)-\rho_{1} \psi(x) \leqslant u_{0}(x) \leqslant u_{e}(x)+\rho_{2} \psi(x) \quad(x \in \bar{\Omega}) .
$$

On the other hand, if

$$
\exp \left(a u_{e}(x)\right)>(a \lambda)^{-1} \mu_{0} \quad(x \in \bar{\Omega}),
$$

then there exists $\alpha_{3}>0$ such that for every positive constant $\rho_{3}$,

$$
u(t, x) \geqslant u_{e}(x)+\rho_{3} e^{\alpha_{3} t} \psi(x) \quad(t>0, x \in \bar{\Omega})
$$

whenever $u_{0}(x) \geqslant u_{e}(x)+\rho_{3} \psi(x)$ for $x \in \bar{\Omega}$.

Remarks. (a) The conclusions in Theorems 1, 2 and 3 remain valid when the operator $\nabla \cdot(D(x) \nabla u)$ is replaced by a uniformly elliptic operator of the form

$$
L u \equiv \sum_{i, j=1}^{n} a_{i j}(x) u_{x_{i} x_{j}}+\sum_{i=1}^{n} a_{i}(x) u_{x_{i}}
$$

In this situation, $\mu_{0}$ and $\psi$ are the least eigenvalue and its corresponding eigenfunction of $L$ (under the boundary condition in (2.1)). Notice that $\mu_{0}$ is real, positive and $\psi(x)$ is positive in $\Omega$ or $\bar{\Omega}$ according to $\beta=0$ or $\beta>0$ (cf. [12], [13]).

(b) Since $\mu_{0}^{-1}$ is directly related to the size of $\Omega$ (for certain fixed geometry). Theorems 1 and 2 imply that for fixed $\lambda$ if we increase the size of $\Omega$ then there is a critical domain $\Omega_{c}$ such that a global solution exists and converges to zero (for small $u_{0}$ and $b=1$ ) when $\Omega$ is smaller than $\Omega_{c}$, and there exists no global solution when $\Omega$ is larger than $\Omega_{c}$. This phenomenon also leads to a bifurcation result in terms of the size of the spatial domain.

3. Proof of the main theorems. The proof of the results in Theorems 1,2 and 3 is based on the notion of upper and lower solution which has frequently been used in the treatment of both elliptic and parabolic type boundary-value problems (cf. [1], [9], [11], [14]). In this paper, by an upper and lower solution we mean a pair of smooth functions $\tilde{u}(t, x)$ and $\underset{\sim}{u}(t, x)$ 
such that for every finite $T>0, \tilde{u} \geqslant \underset{\sim}{u}$ on $[0, T] \times \bar{\Omega}$ and $\tilde{u}$ satisfies the inequalities

$$
\begin{aligned}
\tilde{u}_{t}-\nabla \cdot(D(x) \nabla \tilde{u}) & \geqslant \lambda\left(e^{a \tilde{u}}-b\right) \quad(t \in(0, T], x \in \Omega), \\
\beta(\partial \tilde{u} / \partial \nu)+\tilde{u} & \geqslant 0 \quad(t \in(0, T], x \in \partial \Omega), \\
\tilde{u}(0, x) & \geqslant u_{0}(x) \quad(x \in \Omega),
\end{aligned}
$$

while $\underset{\sim}{u}$ satisfies all the reversed inequalities in (3.1). Here by a smooth function is meant a continuous function $v$ on $[0, T] \times \bar{\Omega}$ which is continuously differentiable in $t>0$, twice continuously differentiable in $x \in \Omega$ and $\partial v / \partial \nu$ exists on. $(0, T] \times \partial \Omega$.

By starting from the initial iterations $u^{(0)}=\tilde{u}$ and $u^{(0)}=\underline{u}$ we can construct two sequences from the linear system

$$
\begin{aligned}
u_{t}^{(k)}-\nabla \cdot\left(D(x) \nabla u^{(k)}\right) & =\lambda\left(\exp \left(a u^{(k-1)}\right)-b\right) \quad(t \in(0, T], x \in \Omega), \\
\beta\left(\partial u^{(k)} / \partial v\right)+u^{(k)} & =0 \quad(t \in(0, T], x \in \partial \Omega), \\
u^{(k)}(0, x) & =u_{0}(x) \quad(x \in \Omega), \quad k=1,2, \ldots
\end{aligned}
$$

Denote these two sequences, respectively, by $\left\{\bar{u}^{(k)}\right\}$ and $\left\{\underline{u}^{(k)}\right\}$. Then by the property of $\tilde{u}$ and $\underline{u}$ it is easily seen that $\left\{\bar{u}^{(k)}\right\}$ is monotone nonincreasing while $\left\{\underline{u}^{(k)}\right\}$ is monotone nondecreasing and $\underline{u}^{(k)} \leqslant \bar{u}^{(k)}$ for every $k=$ $1,2, \ldots$ Thus the pointwise limits of these sequences exist and are denoted by $\bar{u}$ and $\underline{u}$. A regularity argument shows that $\bar{u}$ and $\underline{u}$ are both solutions of (1.1)-(1.3) and satisfy the relations

$$
\underline{u} \leqslant \underline{u}^{(1)} \leqslant \underline{u}^{(2)} \leqslant \cdots \leqslant \underline{u} \leqslant \bar{u} \leqslant \cdots \leqslant \bar{u}^{(2)} \leqslant \bar{u}^{(1)} \leqslant \tilde{u} .
$$

In fact, $\bar{u}$ coincides with $\underline{u}$ and is the unique solution of (1.1)-(1.3). (See [11], [14] for a detailed discussion. See also [10] for the uniqueness problem.) Hence the asymptotic behavior of the solution can be determined through the construction of suitable upper and lower solutions. The proofs of our main theorems are based on some explicit construction of these functions. Before doing this, we observed that $u=0$ satisfies all the reversed inequalities in (3.1) and thus the problem has a unique nonnegative solution if there exists a nonnegative upper solution. On the other hand, if $\underset{\sim}{u}$ is a function satisfying the reversed inequality in (3.1) then the corresponding sequence $\left\{\underline{u}^{(k)}\right\}$ is monotone nondecreasing and thus converges to the unique solution when it is bounded from above. This approach will be used in the proof of the nonexistence of a global solution.

Proof OF THEOREM 1. We seek a lower solution in the form of $\underset{u}{u}=$ $p(t) \psi(x)$ where $p(t)$ is a positive function with $p(0) \geqslant \delta$. To insure that $\underset{\sim}{u}$ satisfies the reversed inequalities in (3.1) it suffices to find $p$ such that

$$
\begin{array}{r}
p^{\prime}(t) \psi(x)+\mu_{0} p(t) \psi(x) \leqslant \lambda[\exp (a p(t) \psi(x))-b] \\
(t \in(0, T], x \in \Omega) .
\end{array}
$$


Since $e^{a \eta}-b \geqslant a \eta+a^{2} \eta^{2} / 2$ for $\eta \geqslant 0$, where $0 \leqslant b \leqslant 1$, we only need to find $p$ such that $p(0) \geqslant \delta$ and

$$
p^{\prime}(t)-\left(a \lambda-\mu_{0}\right) p(t) \leqslant \lambda a^{2} p^{2}(t) \psi(x) / 2 .
$$

In view of the hypothesis $a \lambda \geqslant \mu_{0}$ the above inequality holds if we choose

$$
p(t)=\delta(1-\gamma t)^{-1}, \quad t \in\left[0, \gamma^{-1}\right),
$$

where $\gamma=\lambda \delta a^{2} \psi_{m} / 2$. With this choice of $p$ the function $\underset{\sim}{u}=p(t) \psi(x)$ becomes a lower solution if there exists an upper solution $\tilde{u}$ satisfying $\tilde{u} \geqslant \tilde{\sim}$ on $\left[0, \gamma^{-1}\right) \times \bar{\Omega}$. However, since $\underset{\sim}{u}$ is unbounded at every point of $\bar{\Omega}$ as $t \rightarrow \gamma^{-1}$ such a function cannot exist unless the solution is also unbounded at all points $x \in \bar{\Omega}$ as $t \rightarrow \gamma^{-1}$. To overcome this difficulty we take a sufficiently large constant $N>u_{0}(x)$ and define a function $f$ by $f(\eta)=e^{a \eta}-b$ for $0 \leqslant \eta \leqslant N$ and $f(\eta)=e^{a N}-b$ for $\eta>N$. Choose $T^{\prime}$ sufficiently close to $\gamma^{-1}$ such that $\underset{\sim}{u}\left(T^{\prime}, x^{\prime}\right) \geqslant N$ for some $x^{\prime} \in \bar{\Omega}$. Then the sequence $\left\{\underline{u}^{(k)}\right\}$ obtained from (3.2) with $\exp \left(a u^{(k-1)}\right)-b$ replaced by $f\left(u^{(k-1)}\right)$ and with $u^{(0)}=p \psi, T=T^{\prime}$ is monotone nondecreasing. Since $f\left(u^{(k-1)}\right)$ is uniformly bounded, the well-known estimate for linear parabolic system insures that $\left\{u^{(k)}\right\}$ is bounded (cf. [4, p. 146]). It follows from its nondecreasing property that $\left\{\underline{u}^{(k)}\right\}$ converges pointwise to a function $u^{*}(t, x)$. A regularity argument shows that $u^{*}$ is a solution of the equation

$$
u_{t}-\nabla \cdot(D(x) \nabla u)=\lambda f(u) \quad\left(t \in\left(0, T^{\prime}\right], x \in \Omega\right)
$$

and satisfies conditions (1.2), (1.3). Since $u^{*} \geqslant \underset{\sim}{u} \equiv p \psi$ on $\left[0, T^{\prime}\right] \times \bar{\Omega}$ and $\underset{\sim}{u}\left(T^{\prime}, x^{\prime}\right) \geqslant N$ there exists $T^{*} \leqslant T^{\prime}$ such that $u^{*}(t, x) \leqslant N$ on $\left[0, T^{*}\right] \times \bar{\Omega}$ and $u^{*}\left(T^{*}, x_{0}\right)=N$ for some $x_{0} \in \bar{\Omega}$. By the definition of $f, u^{*}$ is the solution of $(1.1)-(1.3)$ for at least $(t, x) \in\left[0, T^{*}\right] \times \bar{\Omega}$. The above conclusion shows that for every large constant $N$ there is a number $T^{*}<\gamma^{-1}$ and a solution $u^{*}(t, x)$ of (1.1)-(1.3) on $\left[0, T^{*}\right] \times \bar{\Omega}$ such that $\max u^{*}(t, x)=N$. Hence (2.2) must hold. For if $u$ were bounded on $\left[0, \gamma^{-1}\right) \times \bar{\Omega}$, say by $K$, then by the choice of $N>K$ there would exist a point $x_{0} \in \bar{\Omega}$ and $T^{*}<\gamma^{-1}$ such that $u\left(T^{*}, x_{0}\right)=N>K$ which is absurd. This completes the proof of the theorem.

Proof of Theorem 2. It suffices to find a nonnegative upper solution which converges to zero as $t \rightarrow \infty$ since $\underset{\sim}{u}=0$ clearly satisfies the reversed inequalities in (3.1). We seek an upper solution of the form $\tilde{u}=\rho e^{-\alpha t} \psi(x)$ for some positive constants $\rho$ and $\alpha$. In view of (3.1), $\tilde{u}$ is an upper solution if

$$
\left(\mu_{0}-\alpha\right) \rho e^{-\alpha t} \psi(x) \geqslant \lambda\left[\exp \left(a \rho e^{-\alpha t} \psi(x)\right)-1\right]
$$

The above inequality holds if

$$
\left(\mu_{0}-\alpha\right) \eta \geqslant \lambda[\exp (a \eta)-1] \text { for } 0 \leqslant \eta \leqslant \rho .
$$

But by the hypothesis $\lambda a<\mu_{0}$ there exists $\rho>0$ such that

$$
(a \eta)^{-1}\left(e^{a \eta}-1\right)<(\lambda a)^{-1} \mu_{0} \text { for } 0 \leqslant \eta \leqslant \rho .
$$

It follows by letting $\alpha=\mu_{0}-\rho^{-1} \lambda\left(e^{a \rho}-1\right)>0$ that 


$$
\begin{aligned}
(\lambda a)^{-1}\left(\mu_{0}-\alpha\right) & =(a \rho)^{-1}\left(e^{a \rho}-1\right) \\
& \geqslant(a \eta)^{-1}\left(e^{a \eta}-1\right) \quad(0<\eta \leqslant \rho),
\end{aligned}
$$

which proves (3.9). Hence a unique nonnegative solution $u(t, x)$ exists and is bounded by $\rho e^{-\alpha t} \psi(x)$. Relation (2.3) follows immediately.

Proof of Theorem 3. To prove relation (2.5) it suffices to show that $\tilde{u}=u_{e}(x)+\rho_{2} e^{-\alpha_{2} t} \psi(x)$ and $\underset{\sim}{u}=u_{e}(x)-\rho_{1} e^{-\alpha_{1} t} \psi(x)$ are upper and lower solutions, respectively. Clearly, $\tilde{u}$ is an upper solution if

$$
\begin{aligned}
-\nabla \cdot(D(x) & \left.\nabla u_{e}\right)+\left(\mu_{0}-\alpha_{2}\right) \rho_{2} e^{-\alpha_{2} t} \psi(x) \\
& \geqslant \lambda\left[\exp \left(a u_{e}(x)\right) \exp \left(a \rho_{2} e^{-\alpha_{2} t} \psi(x)\right)-b\right]
\end{aligned}
$$

since both $u_{e}$ and $\psi$ satisfy the boundary condition (1.2). From $-\nabla \cdot\left(D \nabla u_{e}\right)$ $=\lambda\left(\exp \left(a u_{e}\right)-b\right)$ the above inequality holds if

$$
\left(\mu_{0}-\alpha_{2}\right) \eta>\lambda\left(\exp \left(a u_{e}(x)\right)\right)[\exp (a \eta)-1] \quad\left(0 \leqslant \eta \leqslant \rho_{2}\right) .
$$

But this follows from hypothesis (2.4) for some sufficiently small $\rho_{2}$ and $\alpha_{2}$. The proof of $\underset{\sim}{u}$ being a lower solution is similar. To show the relation (2.7) we observe that the function $\underset{\sim}{v}=u_{e}(x)+\rho_{3} e^{\alpha_{3} t} \psi(x)$ satisfies the reversed inequalities in (3.1) if

$$
\left(\mu_{0}+\alpha_{3}\right) \eta \leqslant \lambda \exp \left(a u_{e}(x)\right)\left(e^{a \eta}-1\right) \text { for } \eta \geqslant 0 .
$$

In view of (2.6) and the nonnegative property of $u_{e}$ this relation is clearly satisfied by some $\alpha_{3}>0$. Using the modified function $f(\eta)$ and the initial iteration $u^{(0)}=\underset{\sim}{u}$ we conclude from the same argument as in the proof of Theorem 1 that a solution $u(t, x)$ to $(1.1)-(1.3)$ exists and satisfies $u(t, x)>$ $\underset{\sim}{u}(t, x)$ on $[0, T] \times \bar{\Omega}$ for every finite $T$. This completes the proof of the theorem.

REMARK. The proofs of Theorems 1, 2 and 3 yield the following additional information. (i) The finite escape time $T_{1}$ is bounded by $2\left(\lambda \delta a^{2} \psi_{m}\right)^{-1}$ which has a natural physical interpretation. (ii) The constants $\rho, \alpha$ which give the extent of stability and the exponential rate of decay can be explicitly estimated from $\left(\mu_{0}-a \lambda\right)$. (iii) A stability region of $u_{e}(x)$ is contained between $u_{e}(x)-\rho_{1} \psi(x)$ and $u_{e}(x)+\rho_{2} \psi(x)$ where $\rho_{1}, \rho_{2}$ can be estimated from the bound of $u_{e}$ (e.g., see [2] for such a bound).

\section{REFERENCES}

1. H. Amann, On the existence of positive solutions of nonlinear elliptic boundary value problems, Indiana Univ. Math. J. 21 (1971/72), 125-146. MR 45 \# 5558.

2. C. Bandle, Existence theorems, qualitative results and a priori bounds for a class of nonlinear Dirichlet problems, Arch. Rational Mech. Anal. 59 (1975), 219-238.

3. J. P. G. Ewer and L. A. Peletier, On the asymptotic behavior of solutions of semilinear parabolic equations, SIAM J. Appl. Math. 28 (1975), 43-53.

4. A. Friedman, Partial differential equations of parabolic type, Prentice-Hall, Englewood Cliffs, N. J., 1964. MR 31 \#6062.

5. H. Fujita, On the nonlinear equations $\Delta u+e^{u}=0$ and $\partial v / \partial t=\Delta v+e^{v}$, Bull. Amer. Math. Soc. 75 (1969), 132-135. MR 39 \#615. 
6. I. M. Gel'fand, Some problems in the theory of quasilinear equations, Amer. Math. Soc. Transl. (2) 29 (1963), 295-381. MR 27 \#3921.

7. K. Hayakawa, On non-existence of global solutions of some semi-linear parabolic differential equations, Proc. Japan Acad. 49 (1973), 503-505.

8. D. D. Joseph and E. M. Sparrow, Nonlinear diffusion induced by nonlinear sources, Quart. Appl. Math. 28 (1970), 327-342. MR 42 \# 7153.

9. H. B. Keller and D. S. Cohen, Some positone problems suggested by nonlinear heat generation, J. Math. Mech. 16(1967), 1361-1376. MR 35 \#4552.

10. C. V. Pao, Successive approximations of some nonlinear initial boundary value problems, SIAM J. Math. Anal. 5 (1974), 91-102. MR 49 \# 4265.

11. Positive solutions of a nonlinear boundary value problem of parabolic type, J. Differential Equations 22 (1976), 145-163.

12. M. H. Protter and H. F. Weinberger, Maximum principles in differential equations, Prentice-Hall, Englewood Cliffs, N. J., 1967. MR 36 \#2935.

13. On the spectrum of general second order operators, Bull. Amer. Math. Soc. 72 (1966), 251-255. MR 32 \# 7939.

14. D. H. Sattinger, Monotone methods in nonlinear elliptic and parabolic boundary value problems, Indiana Univ. Math. J. 21 (1971/72), 979-1000. MR 45 \#8969.

15. S. Sugitani, On non-existence of global solutions for some nonlinear integral equations, Osaka J. Math. 12 (1975), 45-51.

Department of Mathematics, North Carolina State Univeristy, Raleigh, North Carolina 27607 